\title{
Swelling and Erosion Modulation of Sterculia Foetida Gum Through Real Time Texture Probing
}

\author{
Bendgude Namdeo, lyer Vidya and Poddar Sushilkumar \\ Department of Pharmaceutics, Principal K. M. Kundnani College of Pharmacy, \\ 23 Jote Joy Bldg.Cuffe Parade, Colaba, Mumbai 400005, India
}

\begin{abstract}
In the present investigation an attempt has been made to increase therapeutic efficacy, reduce frequency of administration and improve patient compliance by developing controlled release matrix tablets of diltiazem hydrochloride. Diltiazem hydrochloride was formulated as oral controlled release matrix tablets by using sterculia foetida gum. SFG fines were characterized with scanning electron microscopy. The purpose of this study was to optimize release profile of the highly water soluble drug from SFG matrix by using water soluble and swellable excipients like lactose and microcrystalline cellulose respectively. Tablets were prepared by direct compression, and their swelling behavior in presence of these excipients was assessed with the help of a Texture Analyzer. Dissolution assessment was performed using USP 26 apparatus 2 modified by insertion of a mesh to prevent sticking of the tablets to the bottom of vessel and allow them to swell three dimensionally. The interdependence of swelling front movement in relation to excipients type and progression of drug release are explained. It was concluded that unlike in conventional dosage forms insertion of excipients in hydrophilic controlled release tablets containing a water soluble drug gave the finger print information of drug release profile. In vitro drug release from these matrices was characterized and confirmed with the help of real time texture probing. Results indicated that it is possible to achieve desired modulation in the drug release profile by inclusion of lactose and microcrystalline cellulose.
\end{abstract}

Key words: Diltiazem HCl, Sterculia Foetida Gum, Swelling and erosion, Lactose, Texture analysis.

\section{INTRODUCTION}

The development of controlled release drug delivery system has long been a major area of research in the pharmaceutical industry. Matrix tablet systems appear to be one of the most attractive approaches from an economic as well as from the process development and scale up point of view. The most important concern in these systems is the rate at which the drug substance is released. In the preparation and study of matrix tablets it was thought

Correspondence to: Poddar Sushilkumar Sharatchandra E-mail:ssp306@rediffmail.com

Tel: 022-22164387, 22164368; Fax 022- 22165282

Dhaka Univ. J. Pharm. Sci. 7(2): 127-132, 2008 (December) that there are diverse factors controlling drug release e.g. particle size of drug and excipients, compression force, nature of polymer support and diluents.

Designing of such a delivery system involves the application of polymer chemistry in the dosage form construction to produce well characterized and reproducible units. Such units are able to provide controlled drug delivery in to body within the specification of required drug delivery profile. Of the approaches known for modified/controlled drug release, hydrophilic matrices continue to receive a good degree of attention, as these devices involve requirement of low fabrication cost and reliability of 
release profile. When such a device is exposed to an aqueous medium it may not disintegrate, but rather hydrates and develops a highly viscous gelatinous surface barrier which controls both the liquid penetration into the center of the hydrophilic matrix system and the drug release from the same. The overall release rate of a drug from this system is controlled by one or more of the following processes: transport of the solvent into the unit, swelling of the associated matrix, diffusion of the solute through the swollen matrix, erosion of the swollen matrix etc. ${ }^{1}$ It is noted that many 'old' materials compete successfully even today after almost a century of efforts of replacement by newer materials. While it is a usual balance of economics and performance that determines the commercial realities.

SFG is chemically characterized to be containing high acetyl content, a high proportion of Dgalacturonic acid and a presence of residues of Lrhamnose, D-galactose and a ketohexose. The SFG in the form of compressed tablets when placed in dissolution medium do not disintegrate, but after hydration develop a highly viscous gelatinous surface barrier layer. For this reason, it appeared to be an interesting polymer for the preparation of hydrophilic matrix tablets. In our work, attention was focused on swelling behavior of the SFG with the help of texture analyzer by inclusion of different types of excipients. For this study, Diltiazem Hydrochloride (DIL) was used as a model drug. ${ }^{2}$

\section{MATERIALS AND METHODS}

DIL $\mathrm{HCl}$ was obtained from Themis laboratory Mumbai as a gift sample and SFG from the University Institute of Chemical Technology (UICT) Mumbai. The excipients/fillers lactose monohydrate, microcrystalline cellulose (PH 101), Magnesium stearate and talc were obtained from Evonics India Mumbai. All other reagents used were of analytical grade.

Preparation of matrix tablets. Three different batches of tablets were prepared and referred to as A, B and C. DIL HCl (15\% w/w), SFG (64\% w/w), lactose monohydrate $(19 \% \mathrm{w} / \mathrm{w})$, magnesium stearate $(0.5 \% \mathrm{w} / \mathrm{w})$ and talc $(1.5 \% \mathrm{w} / \mathrm{w})$ constituted formulation $\mathrm{A}$ and microcrystalline cellulose (CMCC) PH $101(19 \% \mathrm{w} / \mathrm{w})$ in place of lactose monohydrate was used in the formulation $\mathrm{B}$. These materials were screened through a sieve of number 80 and mixed together in mortar by using pastel. Final mixtures were compressed into tablets using single punch $(8.5 \mathrm{~mm})$ tablet press. Formulation C containing drug, SFG $(83 \% \mathrm{w} / \mathrm{w})$, magnesium stearate and talc were prepared under identical condition as shown in Table 1.

Table 1. Composition of Diltiazem HCI tablets.

\begin{tabular}{ccccccc}
\hline $\begin{array}{c}\text { Component } \\
\% \text { w/w }\end{array}$ & $\begin{array}{c}\text { Diltia } \\
\text {-zem } \\
\text { HCl }\end{array}$ & SFG & $\begin{array}{c}\text { Lac- } \\
\text { tose }\end{array}$ & MCC & $\begin{array}{c}\text { Mag- } \\
\text { nesium } \\
\text { stearate }\end{array}$ & Talc \\
\hline $\begin{array}{c}\text { Formulation } \\
\text { A }\end{array}$ & 15 & 64 & 19 & 00 & 0.5 & 1.5 \\
$\begin{array}{c}\text { Formulation } \\
\text { B }\end{array}$ & 15 & 64 & 00 & 19 & 0.5 & 1.5 \\
$\begin{array}{c}\text { Formulation } \\
\text { C }\end{array}$ & 15 & 83 & 00 & 00 & 0.5 & 1.5 \\
\hline
\end{tabular}

Stability studies. The accelerated stability studies was done at $40^{\circ} \mathrm{C}$ and $75 \% \mathrm{RH}$ for 4,8 and 12 weeks intervals and subjected to in vitro drug studies. Formulations were stored in amber color screw capped glass bottle at different temperature and humidity condition.

Evaluation of tablets. The prepared matrix tablets were evaluated for hardness, weight variation, thickness, and friability. The hardness of the tablets was tested using Monsanto hardness tester. Friability of tablets was determined in a Roche friabilator. The thickness of tablets was measured by vernier calipers. Weight variation test was performed according to IP method. Tablet dissolution was assessed thrice per batch using USP 26 Apparatus 2 (Paddle) at $50 \mathrm{rpm}$ in distilled water at $37^{\circ} \mathrm{C}$. To avoid the adhesion of the hydrating tablets to the bottom of the dissolution vessel the apparatus was modified by the inclusion of a stainless steel mesh basket device in each vessel. The drug concentration was determined every one hour by Shimadzu UV spectrophotometer at $238 \mathrm{~nm}$.

Scanning Electron Microscopy. A sample of SFG was mounted on a stub (aluminum mount) and 
stuck with colloidal silver liquid adhesive and left to dry. The sample was put in the Auto Sputter Coater (Biorad E5200) and covered with gold layer. Argon gas was used for the vacuum. Each second, $3 \mathrm{~A}^{0}$ of gold was applied on the sample. The photomicrographs were taken at 200x, 350x, and 550x magnifications.

Texture analysis. Texture analysis study of the swollen tablets was done by exposing the tablet in dissolution medium $900 \mathrm{ml}$ at $37^{\circ} \mathrm{C}$ (Distilled water) using USP 26 apparatus 2 (paddle) at $50 \mathrm{rpm}$. To avoid random sticking of tablets to dissolution jar and to facilitate when needed, tablet was stuck initially to a piece of glass slide (about1" $\times 1$ ") with the help of a drop of water. Once the treatment in jar start, tablets with the glass slide should be removed from the dissolution jar after each hour and texture analysis carried out. Texture analysis was performed using TA.2Xi texture analyzer equipped with 500gm load and texture expert software. The forcedisplacement vs. time profiles associated with the penetration of cylindrical stainless steel probe of $2 \mathrm{~mm}$ dia in to the swollen matrices were monitored at data acquisition rate of 250 points per second. Probe approached the sample at pretest speed of $2 \mathrm{~mm} / \mathrm{s}$. Once a trigger force of $0.7 \mathrm{~g}$ was detected (at contact of probe with tablet) probe was advanced into the sample at the test speed of $0.2 \mathrm{~mm} / \mathrm{s}$ until the maximum force at the glassy core boundary was reached.

\section{RESULTS AND DISCUSSION}

The result of hardness and friability of the prepared matrix tablets ranged from 6.5 to $7.0 \mathrm{~kg} / \mathrm{cm}^{2}$ and 0.94 to $0.99 \%$ respectively. Tablet formulations of all the batches having DIL HCl within $100 \pm 5 \%$ of labelled content are given in Table 2.

The drug release from formulation A having the ratio of drug: polymer: lactose as $1: 4.2: 1.3$ showed higher rate than the formulations $\mathrm{B} \& \mathrm{C}$ having the ratio of drug: polymer: MCC as $1: 4.26: 1.33$ and drug: polymer as $1: 5.6$, respectively. The results of dissolution studies of formulation A, B and C are shown in Fig. 5. As the Formulation $\mathrm{C}$ contained higher percentage of polymer it showed a linear i.e. $62.98 \%$ of drug release in 8 hrs. However the formulation A and B having comparatively lower percentage of polymer showed $83.68 \%$ and $72.16 \%$ of drug release respectively within the same period of time. The formulations A, B and C showed linear regression coefficient $(\mathrm{R})$ values 0.9989, 0.9940, 0.9965 , slope (n) value of $0.7570,0.7139,0.7030$ and diffusion rate constant $(\mathrm{K})$ values $15.44,14.41,14.36$ respectively as shown in Table 3. According to these regression coefficient values best fitted model for the formulation A and B was Hixson Crowell and first order for the formulation $\mathrm{C}$. The $\mathrm{n}$ values calculated by generally used exponential Korsemeyer Peppas equation for matrix system appear to indicate a coupling of diffusion and erosion mechanism (known as the anomalous non-fickian diffusion. Hence, diffusion coupled with erosion may be the mechanism of DIL $\mathrm{HCl}$ in this case. Generally lactose is widely used channeling agent and able to give good reproducible results. Batch A was selected for stability studies. Dissolution studies were conducted in distilled water, no significant difference ( $P>0.05)$ was observed in percentage of DIL release from the formulation stored at same condition as compared to the formulation tried before accelerated stability studies. As shown in Table 4.

Table 2. Properties of Diltiazem HCl tablets.

\begin{tabular}{cccccc}
\hline Formulations & $\begin{array}{c}\text { Weight (SD) } \\
\mathrm{mg}(\mathrm{n}=10)\end{array}$ & $\begin{array}{c}\text { Hardness (SD) } \\
\mathrm{kg} / \mathrm{cm}^{2}(\mathrm{n}=5)\end{array}$ & $\begin{array}{c}\text { Thickness } \\
\mathrm{mm}\end{array}$ & $\begin{array}{c}\text { Friability (SD) } \\
\%(\mathrm{n}=5)\end{array}$ & $\begin{array}{c}\text { Content uniformity } \\
(\mathrm{SD}) \%(\mathrm{n}=3)\end{array}$ \\
\hline A & $200.22(0.70)$ & $7.0(0.80)$ & 2.0 & $0.99(0.05)$ & $103.60(1.02)$ \\
B & $200.12(0.86)$ & $6.5(0.88)$ & 2.0 & $0.98(0.07)$ & $100.00(0.93)$ \\
C & $200.00(0.88)$ & $7.0(0.73)$ & 2.0 & $0.94(0.03)$ & $98.73(0.95)$ \\
\hline
\end{tabular}

Note: Figures in the parentheses represent \pm SD $(n=3)$ 
Table 3. Mathematical modeling and mechanism of release of Diltiazem HCl tablets.

\begin{tabular}{|c|c|c|c|c|c|c|}
\hline Formulations & K & $\mathrm{n}$ & $\mathrm{R}$ & $\begin{array}{c}\text { Release (SD) } \\
\%(\mathrm{n}=3)\end{array}$ & Model fitting & Mechanism of drug release \\
\hline A & 15.44 & 0.7570 & 0.9989 & $\begin{array}{l}83.68 \\
(0.96)\end{array}$ & Hix Crow. & Non fickian diffusion \\
\hline B & 14.41 & 0.7139 & 0.9940 & $72.16 \%(0.98)$ & Hix Crow. & Non fickian diffusion \\
\hline $\mathrm{C}$ & 14.36 & 0.7030 & 0.9965 & $\begin{array}{c}62.98 \% \\
(0.96)\end{array}$ & First order & Non fickian diffusion \\
\hline
\end{tabular}

Table 4. Stability studies of tablets of batch A.

\begin{tabular}{lcccc}
\hline Parameters & \multicolumn{4}{c}{ Amber color screw capped bottles at $40^{\circ} \mathrm{C}$ with $75 \% \mathrm{RH}$} \\
\cline { 2 - 5 } Time(weeks) & 0 & 4 & 8 & 12 \\
\hline Drug Content (\%) & 99.98 & 99.72 & 99.15 & 98.99 \\
& $(-0.47)$ & $(0.00)$ & $(-0.36)$ & $(-0.57)$ \\
$\mathrm{T}_{10}(\mathrm{hr})$ & $0.96(-4.20)$ & $0.84(0.00)$ & $0.88(0.72)$ & $0.92(0.00)$ \\
$\mathrm{T}_{50}(\mathrm{hr})$ & $5.87(0.00)$ & $6.14(-2.15)$ & $5.97(1.49)$ & $5.67(0.30)$ \\
$\mathrm{T}_{80}(\mathrm{hr})$ & $9.14(-1.42)$ & $9.61(0.00)$ & $9.24(-0.20)$ & $9.36(1.20)$ \\
$\mathrm{T}_{100}(\mathrm{hr})$ & $11.63(0.00)$ & $11.72(0.57)$ & $11.64(2.17)$ & $11.88(0.50)$ \\
\hline
\end{tabular}

Note: Figures in parenthesis indicate deviation from original.

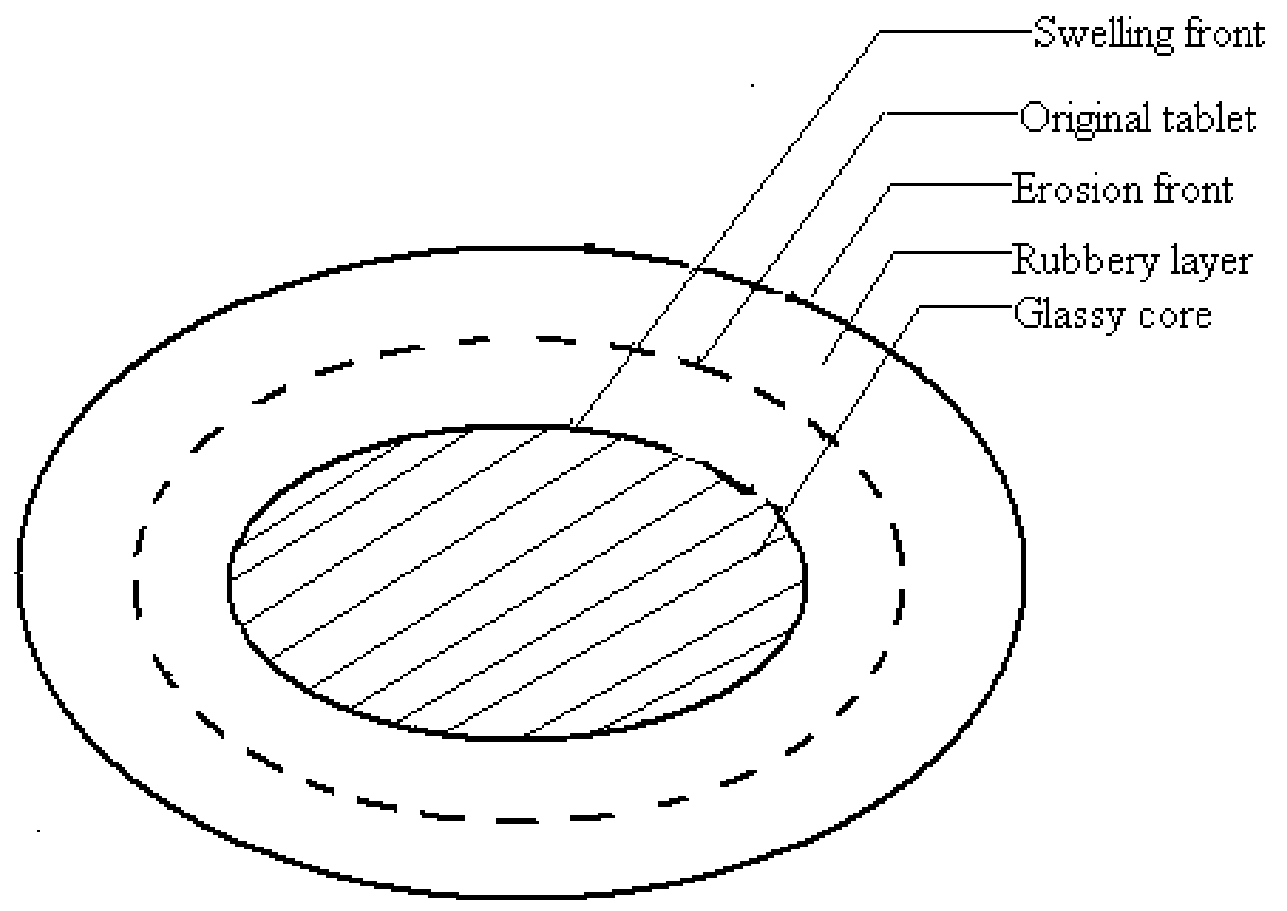

Fig 1. Different layers and fronts during swelling processes of hydrophilic (SFG) polymer.

The release of a drug depends not only on the nature of matrix but also upon the drug polymer ratio. As the percentage of polymer increased, the release rate was noted to be decreasing as shown in Table 3. This may be due to the increase in concentration of SFG resulting in to increase in tortuosity of resulting gel. $^{3}$ Generally polymer dissolution or erosion takes place in three steps viz. 1 . Solvent penetration into the polymer matrix, 2. Polymer swelling and chain disentanglement 3. Attainment of threshold disentanglement. When water penetrates in to polymer matrix, it enhances polymer chain mobility, which eventually disentangles at the advancing front, 
separating the gel layer from the erosion/dissolution front. Polymer swelling occurs as a result of osmotic stress exerted at the advancing glassy core. ${ }^{3}$ Effect is more pronounced in case of inclusion of water soluble drug and/or excipients. The formation of various layers and fronts during the swelling of SFG is shown in Fig. 1.
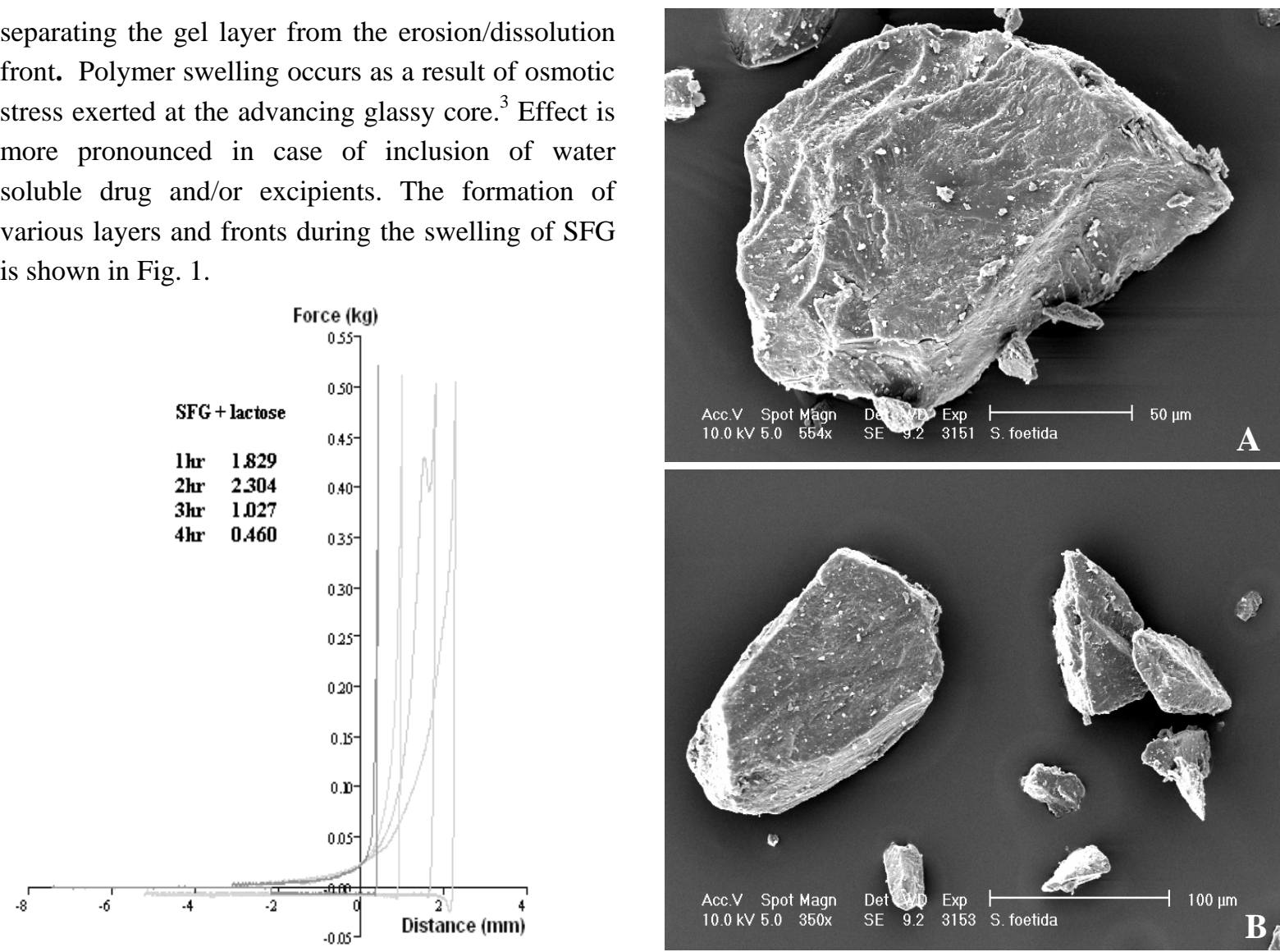

Fig 2. Texture profile showing Probe displacement values for tablet of batch A.

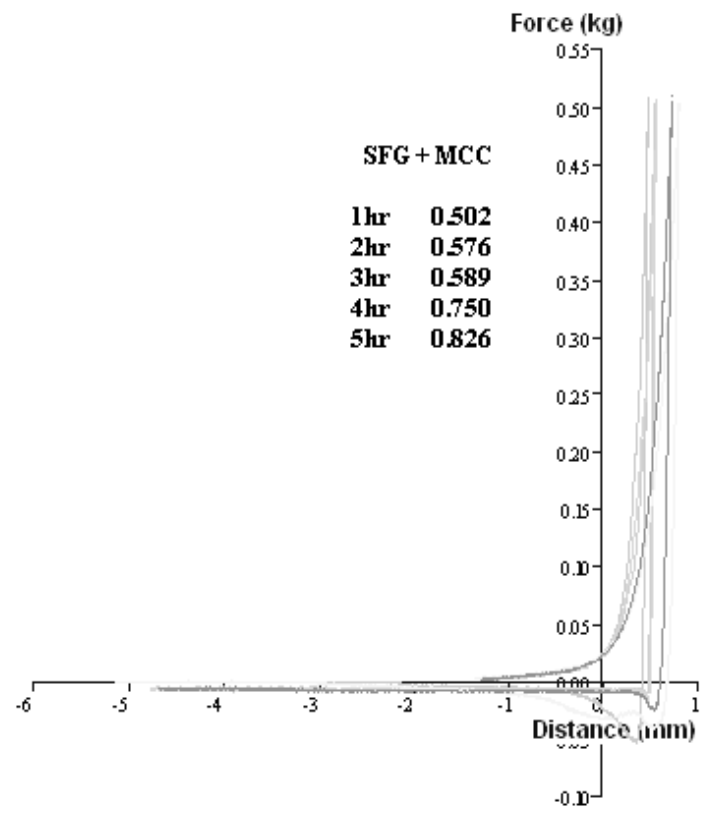

Fig 3. Texture profile showing Probe displacement values for tablet of batch $\mathrm{B}$.
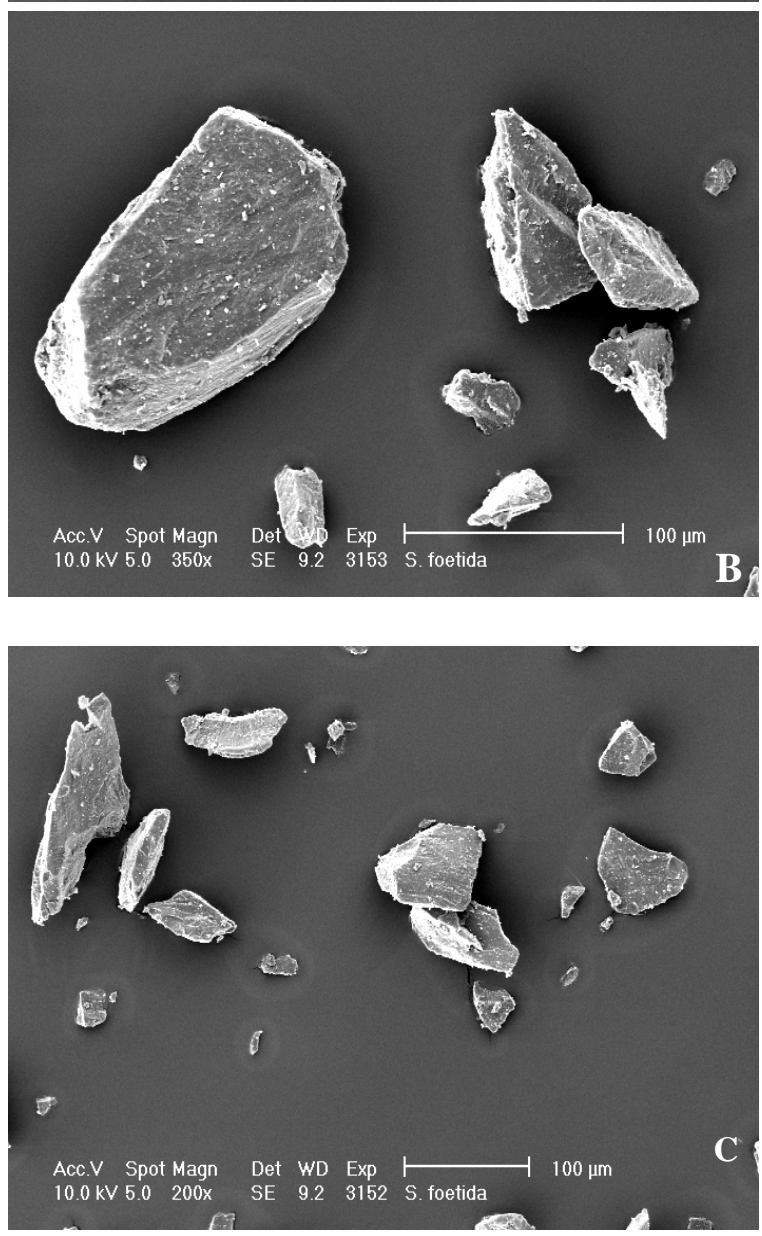

Fig 4. Scanning electron microscopy of sterculia foetida gum powder at three different magnifications (A.554x, B. 350x and C. 200x).

Results indicated that there is initial high probe displacement due to greater hydration rate as noted in readings 0 to1h. Fig 2 shows that the initial sharp increase in displacement with decrease in displacement at latter hydration stage is due to inclusion of water soluble excipients into the 
formulation containing highly soluble drug; this might have been attributed to gradual swelling as well as erosion of tablet matrix over the time. Lactose is water soluble excipient and helps more water to penetrate in to polymer, creates excessive osmotic force and polymer chain relaxation. ${ }^{4}$ Therefore leading to a decrease in tortuosity and/or increase in the matrix porosity. Fig 3 shows a steady increase in displacement value., ${ }^{4,5}$ This is because of inclusion of water swellable excipients microcrystalline cellulose that swells progressively with continuous absorption of water from dissolution medium. Microcrystalline cellulose containing matrices gives more elegant formulation than the lactose containing formulation due to its better direct compression properties, ability to form cohesive compacts, which may help to prolong the release rate. It also acts as a disintegrating agent in tablet formulation.

Surface morphology for the powder particles of sterculia foetida gum was examined by the Scanning Electron Microscope (Philips XL30) as shown in Fig 4. It is observed that pictures support the smoothness of SFG particles at three different magnification levels, which help to develop better cohesion force during tablet compression.

\section{CONCLUSION}

Sterculia foetida gum containing water-soluble drug and excipients demonstrated good swelling and erosion properties as they are certainly capable of altering water penetration and hence mechanism of drug release. The role of gel layer and its rate of growth are central and fundamental to define various fronts and understand the operating release mechanism. The texture analysis method used for studying the dynamics of front movements offers new opportunities to better understand the nature of the solute transport in these systems when substances of different solubility are used. The TA would provide additional operational tool to formulation scientists, allowing them to select optimal and easy to adapt methodology for dosage form development and assessment. Moreover introduction of sterculia foetida gum as a novel natural hydrophilic polymer in controlled release formulations and various interpretations presented may be useful for the pharmaceutical scientists who are busy with designing of hydrophilic matrix systems.

\section{ACKNOWLEDGEMENT}

The authors are thankful to Themis for giving gift sample of DIL $\mathrm{HCl}$; Department of Pharmacognosy and Phytochemistry UICT Mumbai for supplying SFG; Evonics India for providing other excipients. Authors also thankful to Scientific and Digital System, New Delhi for assisting in the texture analysis and CITRON for providing facility for SEM study.

\section{REFERENCES}

1. Pillay, V. and Fassihi, R. 2000. A novel approach for constant rate delivery of highly soluble bioactives from a simple monolithic system. J. Control. Release. 67, 67-78.

2. Chiwate, A., Shajahan, A., Savant G. and Poddar, S.S. 2008. Evaluation of Sterculia Foetida Gum as Controlled release Excipient AAPS Pharm. Sci. Tech. 9, 197-204.

3. Colombo, P., Santi, P., Bettini, R., Brazel, C.S. and Peppas, N.A. 2000. Drug release from swelling-controlled systems. In: Wise, D.L. (Ed.), Handbook of Pharmaceutical Controlled Release Technology. Marcel Dekker Inc, New York, Chapter 9, pp. 183-209.

4. Jamzad, S., Tutunji, L. and Fassihi, R. 2005. Analysis of macromolecular changes and drug release from hydrophilic matrix systems. Int J. Pharm. 292, 75-85.

5. Sami, N., Mahmoud N.B. and Yasser El-Malah. 2007. A novel texture-probe for the simultaneous and real-time measurement of swelling and erosion rates of matrix tablets. Int J. Pharm. 330, 195-198. 